\title{
Exclusive Monte Carlo modelling of NLO DGLAP evolution
}

\author{
S. Jadach ${ }^{* \dagger}$ \\ H. Niewodniczański Institute of Nuclear Physics, Polish Academy of Sciences, \\ ul. Radzikowskiego 152, 31-342 Cracow, Poland \\ E-mail: Stanislaw.Jadach@ifj.edu.pl
}

\section{Skrzypek}

H. Niewodniczański Institute of Nuclear Physics, Polish Academy of Sciences, ul. Radzikowskiego 152, 31-342 Cracow, Poland

E-mail: Maciej.Skrzypek@ifj.edu.pl

\author{
A. Kusina \\ H. Niewodniczański Institute of Nuclear Physics, Polish Academy of Sciences, \\ ul. Radzikowskiego 152, 31-342 Cracow, Poland \\ E-mail: Aleksander.Kusina@ifj.edu.pl

\section{Slawinska} \\ H. Niewodniczański Institute of Nuclear Physics, Polish Academy of Sciences, \\ ul. Radzikowskiego 152, 31-342 Cracow, Poland \\ E-mail: Magdalena.Slawinska@ifj.edu.pl
}

The next-to-leading order (NLO) evolution of the parton distribution functions (PDFs) in QCD is a common tool in the lepton-hadron and hadron-hadron collider data analysis. The standard NLO DGLAP evolution is formulated for inclusive (integrated) PDFs and done using inclusive NLO kernels. We report here on the ongoing project, called KRKMC, in which NLO DGLAP evolution is performed for the exclusive multiparton (fully unintegrated) distributions (ePDFs) with the help of the exclusive kernels. These kernels are calculated within the two-parton phase space for the non-singlet evolution, using Curci-Furmanski-Petronzio factorization scheme. The multiparton distribution, with multiple use of the exclusive NLO kernels, is implemented in the Monte Carlo program simulating multi-gluon emission from single quark emitter. High statistics tests $\left(\sim 10^{10}\right.$ events) show that the new scheme works perfectly well in practice and, at the inclusive (integrated) level, is equivalent with the traditional inclusive NLO DGLAP evolution. Once completed, this new technique is aimed as a building block for the new more precise NLO parton shower Monte Carlo, for W/Z production at LHC and for ep scattering, as well as a starting point for other perturbative QCD based Monte Carlo projects.

RADCOR 2009 - 9th International Symposium on Radiative Corrections (Applications of Quantum Field Theory to Phenomenology),

October 25 - 302009

Ascona, Switzerland

\footnotetext{
* Speaker.

${ }^{\dagger}$ This work is partly supported by the EU Framework Programme grants MRTN-CT-2006-035505 and by the Polish Ministry of Science and Higher Education grants No. 1289/B/H03/2009/37 and 153/6.PR UE/2007/7.
} 
We report on the ongoing effort on the exclusive Monte Carlo (MC) modeling of DGLAP[1] evolution at the NLO level using work of Curci Furmanski and Petronzio (CFP) [2] as a guide and reference.

The so-called factorization theorems $[3,4,5]$ in Quantum Chromodynamics (QCD) are stating that in the high energy scattering process of hadrons, with an experimentally identifiable single large scale (effective mass, transverse momentum etc.) one may reorganize the infinite order perturbative expansion in terms of Feynman diagrams, such that all collinear (mass) singularities are encapsulated into certain well defined objects, called parton distribution functions (PDFs) or parton fragmentation functions (PFFs or jets), while the remaining part, free of such singularities, forms the so-called hard process part (coeff. function). The soft singularities due to zero mass gluon emissions are shown not to disturb or invalidate this picture [4], if they are averaged/integrated over the phase space and properly combined with the virtual contributions. In the physical gauge the PDF/PFF part consists of a well defined Feynman diagrams with the ladder topology. In the early stage of formulating practical QCD perturbative methodology it was found that the most economical way of dealing with the PDF/PFF parts of the process was to define them as inclusive as possible, integrating over transverse momenta and summing up over all partons emitted from the ladder, keeping control only on the total energy (light-cone variable) of the parton entering the hard process, and its type. Such inclusive (collinear) PDF is widely used until today in most of practical QCD calculations, especially for the initial hadrons.

The only exception is the so-called parton shower Monte Carlo (PSMC), where one gains access to all momenta and other quantum numbers in PDF/PFF (ladder) part of the hadronic scattering, for every incoming hadron or outgoing jet. Originally the main role of PSMCs was to describe hadronization of the partons, but they have gradually absorbed the leading order perturbative QCD (pQCD) description [6,7] of the ladders (PDFs, PFFs). With the growing sophistication of the high energy (HE) experimental detectors PSMC became indispensable for understanding data in any modern experiment. However, although pQCD calculations using inclusive PDFs have evolved enormously in their sophistication (evolution of PDFs at NLL, NNLL level, corrections to hard process at NLO, NNLO, new ingenious methods of calculating tree-level multiple parton distributions and more) the PSMCs have stayed, from the $\mathrm{PQCD}$ point of view, where they were 25 years ago, that is at the (improved) LO/LL level, until today! This lack of the progress is not fully understood, but most likely the main reason was that computers fast enough were not available and due to the difficulties in reformulating QCD factorization theorems into a form suitable for stochastic simulation (MC) methods.

We are reporting on the first serious attempt to upgrade parton shower MC for a single incoming quark (non-singlet PDF) to the level of the complete $\mathrm{NLO}^{1}$. This will be done, as in early days of pQCD, in the physical gauge, including first order real and virtual corrections to the basic ladder describing LO level showering of one incoming quark. Our Monte Carlo implementation of NLO DGLAP evolution is: (1) based firmly on Feynman diagrams and standard LIPS, (2) based rigorously on the collinear factorization (eg. EGMPR [3]), (3) implementing exactly NLO $\overline{M S}$ DGLAP evolution at the inclusive level, (5) defining fully unintegrated exclusive ePDFs (the integrand of inclusive PDFs), (6) performing NLO evolution by the MC itself (no use of backward evolution[6]).

\footnotetext{
${ }^{1}$ See also refs. $[8,9,10]$ for similar effort in this direction.
} 


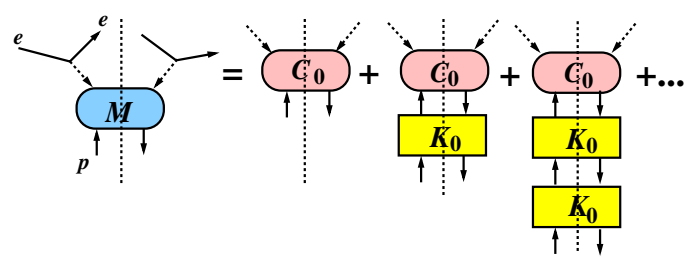

Figure 1: Raw factorization theorem in the physical gauge.

Factorization scheme of EGMPR [3], see Fig. 1, was customized to $\overline{M S}$ by CFP [2]:

$$
\begin{aligned}
& F=C_{0} \cdot \frac{1}{1-K_{0}}=C\left(\alpha, \frac{Q^{2}}{\mu^{2}}\right) \otimes \Gamma\left(\alpha, \frac{1}{\varepsilon}\right), \\
& \Gamma\left(\alpha, \frac{1}{\varepsilon}\right) \equiv\left(\frac{1}{1-K}\right)_{\otimes}=1+K+K \otimes K+K \otimes K \otimes K+\ldots, \\
& K=\mathbb{P} K_{0} \cdot \frac{1}{1-(1-\mathbb{P}) \cdot K_{0}}, \quad C=C_{0} \cdot \frac{1}{1-(1-\mathbb{P}) \cdot K_{0}},
\end{aligned}
$$

where the ladder part $\Gamma$ corresponds to MC parton shower and $C$ is the hard process part ${ }^{2}$. NLO kernels were extracted in ref. [2] from the coefficient of a single pole $\frac{1}{\varepsilon}$ in $\Gamma$. Projection operator of CFP, $\mathbb{P}=P_{\text {spin }} P_{\text {kin }} P P$, consists of the kinematic (on-shell) projection operator $P_{k i n}$, spin projection operator $P_{s p i n}$ and $P P$ extracting pole part $\frac{1}{\varepsilon^{k}}, k>0$.

In our MC solutions we use the standard interpretation [11] of the collinear $\varepsilon$-poles:

$$
\frac{1}{\varepsilon}=\int_{0}^{\mu_{F}} \frac{d k^{T}}{k^{T}}\left(\frac{k^{T}}{\mu_{F}}\right)^{\varepsilon} .
$$

However, the ladder part in CFP/EGMPR scheme features enormous cancellations, as can be seen already at the LO level ${ }^{3}$ :

$$
\Gamma \simeq \frac{1}{1-\left(1-e^{-\frac{1}{\varepsilon}}\right)}=1+\left(1-e^{-\frac{1}{\varepsilon}}\right)+\left(1-e^{-\frac{1}{\varepsilon}}\right)^{2}+\ldots,
$$

while from RGE and explicit LO calculation we obtain readily $\Gamma=e^{+\frac{1}{\varepsilon}}=1+\frac{1}{\varepsilon}+\frac{1}{2 !} \frac{1}{\varepsilon^{2}}+\ldots$ In the MC we need this exponent manifestly, if possible directly from the Feynman diagrams!

The above exponential nature of the QCD evolution of PDFs is manifest in the following master formula

$$
\begin{aligned}
& F=C_{0} \cdot \frac{1}{1-K_{0}}=C_{0} \cdot \overleftarrow{\mathbb{R}}_{\mu}\left[K_{0}\right] \cdot \exp _{T O}\left(\overleftarrow{\mathbb{P}}^{\prime}\left\{{ }^{s} K_{0} \cdot \overleftarrow{\mathbb{R}}_{s}\left[K_{0}\right]\right\}\right)_{\mu} \\
& \overleftarrow{\mathbb{R}}_{\mu}\left(K_{0}\right)=\overleftarrow{\mathbb{B}}_{\mu}\left[\frac{1}{1-K_{0}}\right] \equiv 1+\overleftarrow{\mathbb{B}}_{\mu}\left[K_{0}\right]+\overleftarrow{\mathbb{B}}_{\mu}\left[K_{0} \cdot K_{0}\right]+\overleftarrow{\mathbb{B}}_{\mu}\left[K_{0} \cdot K_{0} \cdot K_{0}\right]+\ldots
\end{aligned}
$$

which is serving as a generating functional of the exclusive parton distributions implemented in the MC. Here, $\exp _{T O}$ is the time ordered exponential in the time evolution variable $t=\ln \mu$, where $\mu$ is factorization scale variable. Operator $\overleftarrow{\mathbb{B}}$ is defined recursively ${ }^{4}$ :

$$
\begin{aligned}
& \overleftarrow{\mathbb{B}}_{\mu}\left[K_{0}\right]=K_{0}-\mathbb{P}_{\mu}^{\prime}\left\{K_{0}\right\}, \\
& \overleftarrow{\mathbb{B}}_{\mu}\left[K_{0} \cdot K_{0}\right]=K_{0} \cdot K_{0}-\mathbb{P}_{\mu}^{\prime}\left\{{ }^{s_{2}} K_{0}\right\} \cdot \mathbb{P}_{s_{2}}^{\prime}\left\{{ }^{s_{1}} K_{0}\right\}-\mathbb{P}_{\mu}^{\prime}\left\{{ }^{s_{2}} K_{0} \cdot \overleftarrow{\mathbb{B}}_{s_{2}}\left[K_{0}\right]\right\}-\overleftarrow{\mathbb{B}}_{\mu}\left[K_{0}\right] \cdot \mathbb{P}_{\mu}^{\prime}\left\{K_{0}\right\}, \\
& \overleftarrow{\mathbb{B}}_{\mu}\left[K_{0} \cdot K_{0} \cdot K_{0}\right]=K_{0} \cdot K_{0} \cdot K_{0}-\mathbb{P}_{\mu}^{\prime}\left\{{ }^{s_{3}} K_{0}\right\} \cdot \mathbb{P}_{s_{3}}^{\prime}\left\{{ }^{s_{2}} K_{0}\right\} \cdot \mathbb{P}_{s_{2}}^{\prime}\left\{{ }^{s_{1}} K_{0}\right\}-\ldots
\end{aligned}
$$

${ }^{2}$ Multiplication symbol $\cdot$ means full phase space integration $d^{n} k$ while convolution $\otimes$ only the integration over the 1-dim. lightcone variable.

${ }^{3}$ Omitting for simplicity $1 / \varepsilon^{n}$ poles due to running of the coupling constant from the consideration.

${ }^{4}$ Similarly as $\beta$-functions in Yennie-Frautschi-Suura[12] subtraction scheme. See also [13]. 
More terms in the recursion can be obtained by expanding eq. (4) in powers of $K_{0}$. The key point is the definition of modified projection operator $\overleftarrow{\mathbb{P}}^{\prime}$ : (a) it does spin projection as $\mathbb{P}$ of CFP, (b) it sets its incoming momentum on-shell in the part of the diagram towards the hard process, (c) it acts on the integrand of the Lorentz invariant phase space (LIPS), before integration, (d) it sets upper limit $\mu$ on the phase space for all its own real (cut) partons, eg. $\mu>s\left(k_{1}, . ., k_{n}\right)=\max \left(k_{i}^{T}\right)$, (e) our preferred choice is rapidity ordering choice; $s\left(k_{1}, . ., k_{n}\right)=a\left(k_{1}, . ., k_{n}\right)=\max \left(k_{i}^{T} / \alpha_{i}\right), \alpha_{i}=k_{i}^{+} / E$, (f) $\overleftarrow{\mathbb{P}}_{\mu}^{\prime}(A)$ acts on $A$ which is at most single$\log$ (collinear) divergent and extracts this singularity from the LIPS integrand ${ }^{5},(\mathrm{~g}) \overleftarrow{\mathbb{P}}_{\mu}^{\prime}\left(K_{0}\right)$ is legal, as $K_{0}$ is single-log divergent, (h) nesting like $\overleftarrow{\mathbb{P}}^{\prime}\left[K_{0} \cdot\left(1-\overleftarrow{\mathbb{P}}^{\prime}\left(K_{0}\right)\right)\right]$ is allowed, as long as its argument is no more than single-log divergent, (e) $\overleftarrow{\mathbb{P}}^{\prime}$ does not include PP operation. Finally, the time ordered exponential reads:

$$
\exp _{T O}\left(\mathbb{P}_{\mu}^{\prime}\{A\}\right)_{\mu}=1+\mathbb{P}_{\mu}^{\prime}\{A\}+\mathbb{P}_{\mu}^{\prime}\left\{{ }^{s_{2}} A\right\} \cdot \mathbb{P}_{s_{2}}^{\prime}\left\{{ }^{s_{1}} A\right\}+\mathbb{P}_{\mu}^{\prime}\left\{{ }^{s_{3}} A\right\} \cdot \mathbb{P}_{s_{3}}^{\prime}\left\{{ }^{s_{2}} A\right\} \cdot \mathbb{P}_{s_{2}}^{\prime}\left\{{ }^{s_{1}} A\right\}+\ldots
$$

where notation $\left\{{ }^{s} A\right\}$ means that $s=a\left(a_{1}, \ldots, a_{n}\right)=\max \left(a_{1}, \ldots, a_{n}\right)$. For instance for $n=3$ the entire integrand multiplied by $\theta_{\mu>s_{3}>s_{2}>s_{1}}$. Variable $\mu$ is constant, while $s_{i}$ depend on the 4-momenta integration variables.

Master formula of eq. (4) is very important for us, as it serves as a generating functional of the exclusive distributions beyond LO implemented in the MC. It should be also stressed that in CFP work the time ordered exponential is present, however, only for the inclusive PDFs. It is obtained there with help of renormalization group equations, not directly from Feynman diagrams. The question of deriving eq. (4) directly from diagrams at any perturbative order remains an open important problem.

From now on, in the factorization formula (4), we focus on the exclusive PDF (ePDF) $\mathscr{D}\left(\mu, k_{1}, \ldots, k_{n}\right)$, which is the integrand of the inclusive PDF:

$$
D(\mu)=\exp _{T O}\left(\overleftarrow{\mathbb{P}}^{\prime}\left\{{ }^{s} K_{0} \cdot \overleftarrow{\mathbb{R}}_{S}\left[K_{0}\right]\right\}\right)(\mu)=\exp _{T O}(K)
$$

The $x$-dependent PDFs (inclusive) are obtained by means of inserting $\delta\left(x-x\left(k_{1}, \ldots, k_{n}\right)\right)$ in the integrand ${ }^{6}$, $D(\mu) \rightarrow D(\mu, x)=D(\mu)_{x}$.

The standard inclusive PDF is obtained form ePDF by integration $D(\mu, x)=\int d \operatorname{Lips} \mathscr{D}\left(\mu, k_{1}, \ldots, k_{n}, x\right)$. It obeys by construction the ordinary evolution equation

$$
\partial_{\mu} D(\mu, x)=\mathcal{P} \otimes D(\mu, x)
$$

with the inclusive DGLAP kernel

$$
\mathcal{P}(x)=\frac{\partial}{\partial \ln (\mu)}\left(K_{\mu}\right)_{x}=\int d \operatorname{Lips} \delta\left(x-\frac{\sum k_{i}^{+}}{E_{0}}\right) \delta\left(1-\frac{s}{\mu}\right) \frac{d}{d \operatorname{Lips}} \overleftarrow{\mathbb{P}}_{\mu}^{\prime}\left\{{ }^{s} K_{0} \cdot \overleftarrow{\mathbb{R}}_{s}\left[K_{0}\right]\right\}
$$

The LO and NLO truncations of the evolution kernel $K_{\mu}$ are: $K_{\mu}^{L O}=\overleftarrow{\mathbb{P}}_{\mu}^{\prime}\left\{{ }^{s} K_{0}\right\}$, taken at $\mathscr{O}\left(\alpha^{1}\right)$ and $K_{\mu}^{N L O}=\overleftarrow{\mathbb{P}}_{\mu}^{\prime}\left\{s\left(K_{0}+K_{0} \cdot\left(1-\overleftarrow{\mathbb{P}}^{\prime}\right) \cdot K_{0}\right)\right\}$, truncated at $\mathscr{O}\left(\alpha^{2}\right)$. The 2PI kernel $K_{0}$ of CFP scheme (nonsinglet bremsstrahlung) at $\mathrm{LO}+\mathrm{NLO}$ is:

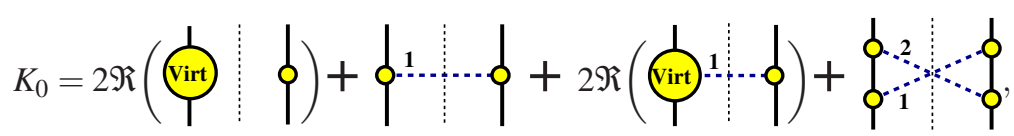

where dashed lines are gluons, blobs marked "Virt" may include several (one loop) subgraphs. First two terms in the $x$-dependent T.O. exponential with LO kernel read

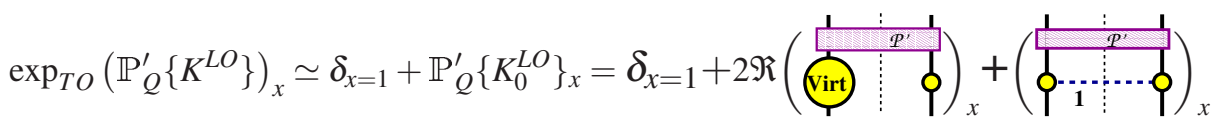

$$
\begin{aligned}
& =\delta_{x=1}+\ln \frac{Q}{q_{0}} \frac{2 C_{F} \alpha_{s}}{\pi}\left(\frac{1+x^{2}}{2(1-x)}\right)_{+}=\delta_{x=1}+\ln \frac{Q}{q_{0}} \mathcal{P}_{q q}(x),
\end{aligned}
$$

\footnotetext{
${ }^{5}$ For instance by rescaling all $k_{i}^{T} \rightarrow \lambda k_{i}^{T}$ and taking coefficient in front of $1 / \lambda$ term.

${ }^{6}$ Such an insertion we shall often mark as $(\ldots)_{x}$. In the MC it means histogramming of $x$.
} 
where $q_{0}$ is IR cut-off and $\mathcal{P}_{q q}(x)=\frac{2 C_{F} \alpha_{s}}{\pi}\left(\frac{1+x^{2}}{2(1-x)}\right)_{+}$.

The LO exclusive distribution $\bar{\rho}_{B 1 r}=\frac{2 C_{F} \alpha_{s}}{\pi^{2}} \frac{1+\left(1-\alpha_{1}\right)^{2}}{2} \frac{1}{\mathbf{k}_{1}^{2}} \theta_{\alpha_{1}>\delta}$ resides inside

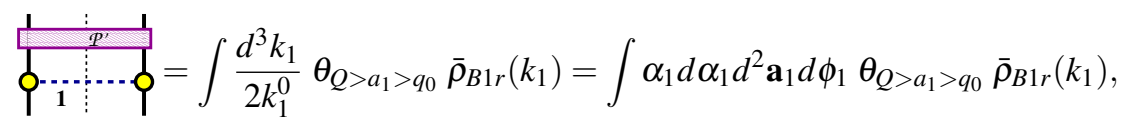

where $\mathbf{a}_{i} \equiv \mathbf{k}_{1} / \alpha_{1}$ and $a_{1}=\left|\mathbf{a}_{1}\right| \simeq$ polar angle of the gluon with transverse momentum $\mathbf{k}_{1}$. The trivial phase space integration gives Sudakov double log or LO kernel:

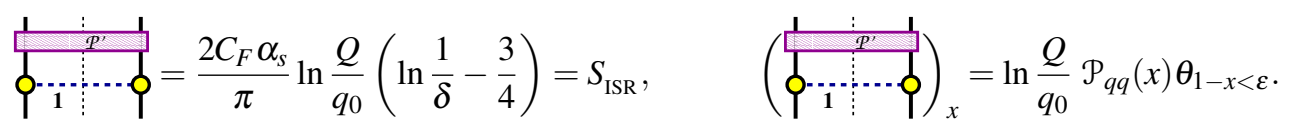

In the TO exponential $D(Q)=\exp _{T O}\left(\mathbb{P}_{Q}^{\prime}\left\{K^{L O}\right\}\right)$ the virtual contribution

$$
\begin{aligned}
& D(Q)=1+\mathbb{P}_{Q}^{\prime}\left\{K_{0}\right\}+\mathbb{P}_{Q}^{\prime}\left\{{ }^{s_{2}} K_{0}\right\} \cdot \mathbb{P}_{s_{2}}^{\prime}\left\{{ }^{s_{1}} K_{0}\right\}+\mathbb{P}_{Q}^{\prime}\left\{{ }^{s_{3}} K_{0}\right\} \cdot \mathbb{P}_{s_{3}}^{\prime}\left\{{ }^{s_{2}} K_{0}\right\} \cdot \mathbb{P}_{s_{2}}^{\prime}\left\{{ }^{s_{1}} K_{0}\right\}+\ldots
\end{aligned}
$$

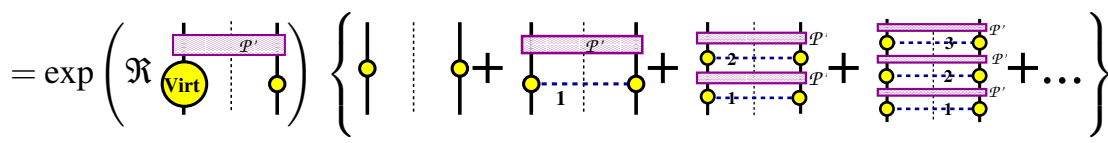

$$
\begin{aligned}
& =e^{-S_{\mathrm{ISR}}}\left\{1+\sum_{n=1}^{\infty} \prod_{j=1}^{n} \int \frac{d^{3} k_{j}}{2 k_{j}^{0}} \Pi\left(k_{j}\right) \bar{\rho}_{B 1}^{L O}\left(k_{j}\right) \theta_{a_{j}>a_{j-1}}\right\}=e^{-S_{\mathrm{ISR}}}\left\{e^{+S_{\mathrm{ISR}}}\right\}=1 .
\end{aligned}
$$

The $x$-dependent version of the same $D(Q, x)=\exp _{T O}\left(\mathbb{P}_{Q}^{\prime}\left\{K^{L O}\right\}\right)_{x}$ reads

$$
D(x, Q)=e^{-S_{\mathrm{ISR}}}\left\{\delta_{x=1}+\sum_{n=1}^{\infty}\left(\prod_{j=1}^{n} \int_{q_{j-1}}^{Q} \frac{d a_{j}}{a_{j}} \int_{0}^{1-\delta} d z_{j} \int_{0}^{2 \pi} \frac{d \varphi_{j}}{2 \pi} \mathcal{P}_{q q}^{\theta}\left(z_{j}\right)\right) \delta_{x=\prod_{i} z_{i}}\right\}
$$

From now on we enter NLO world. The NLO kernel $K_{\mu}^{N L O}=\overleftarrow{\mathbb{P}}_{\mu}^{\prime}\left\{s\left(K_{0}+K_{0} \cdot\left(1-\overleftarrow{\mathbb{P}}^{\prime}\right) \cdot K_{0}\right)\right\}$ with $K_{0}$ of eq. (10) (nonsinglet bremsstrahlung) is inserted into T.O. exponent of the NLO ePFD:

$$
\begin{aligned}
& D_{B}^{[1]}(Q)=\exp \left(-S_{I S R}^{[1]}\right)\left(1+\mathbb{P}_{Q}^{\prime}\left\{K^{r}\right\}+\mathbb{P}_{Q}^{\prime}\left\{{ }^{a_{2}} K^{r}\right\} \cdot \mathbb{P}_{a_{2}}^{\prime}\left\{{ }^{a_{1}} K^{r}\right\}+\right. \\
& \left.+\mathbb{P}_{Q}^{\prime}\left\{{ }^{a_{3}} K^{r}\right\} \cdot \mathbb{P}_{a_{3}}^{\prime}\left\{{ }^{a_{2}} K^{r}\right\} \cdot \mathbb{P}_{a_{2}}^{\prime}\left\{{ }^{a_{1}} K^{r}\right\}+\ldots\right)
\end{aligned}
$$

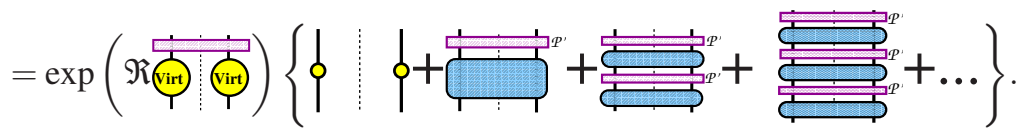

Where the zero-real-emission part of the kernel $-S_{I S R}^{[1]}=$ iiri (wave function renormalization up to second order) factorizes and exponentiates.

The remaining part of the NLO kernel $K^{r}=$ emission part including NLO virtual one loop corrections $K^{1 r}=\phi_{i}$

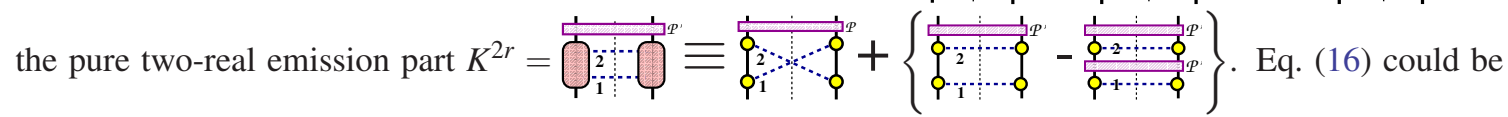
used directly in the MC, if both $K^{1 r}$ and $K^{2 r}$ were positive. Since $K^{2 r}$ is non-positive we have to recombine $K^{2 r}$ with 2 real emission distribution, that is to put it back where it came from (reversing what the 


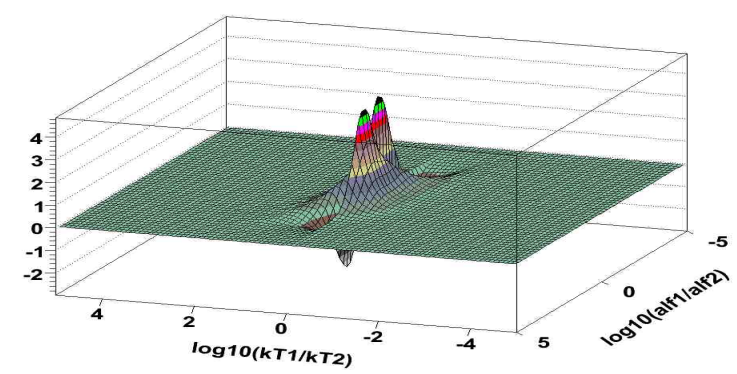

Figure 2: Two-real-gluon NLO correlation function.

factorization did):

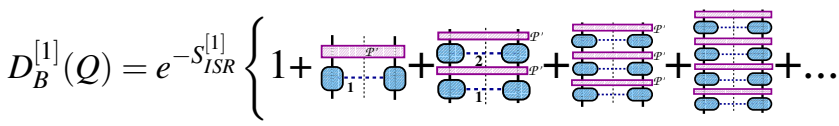

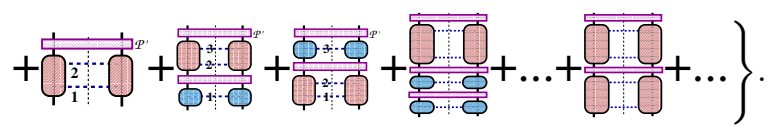

In eq. (17) the first row is positive and large (LO + virtual NLO), hence it will go to basic MC, while the second row being small and nonpositive can be absorbed into MC correcting weight. However, it is not so easy to recombine $K^{2 r}$ with 2 real emissions. The target distribution implemented in the (17) by means of MC-reweighting sero outside simplex $Q>a_{2}>a_{1}>q_{0}$, while the target distribution is nonzero in the bigger rectangle $Q>\max \left(a_{2}, a_{1}\right)>q_{0}$. Going back to original Feynman diagrams we see that the above problem turns out to be fictitious, if we properly keep track of the Bose-Einstein (BE) symmetrization:

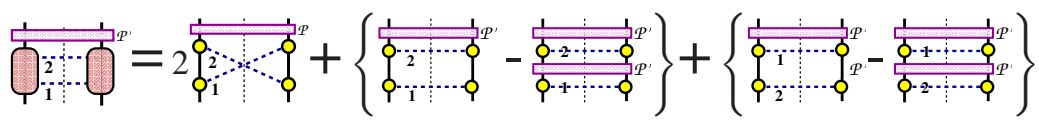

$$
\begin{aligned}
& =\int \frac{d^{3} k_{2}}{2 k_{2}^{0}} \int \frac{d^{3} k_{1}}{2 k_{1}^{0}} \theta_{Q>\max \left(a_{2}, a_{1}\right)>0} \theta_{a_{2}>a_{1}}\left(\beta_{1 B}\left(k_{2}, k_{1}\right)+\beta_{1 B}\left(k_{1}, k_{2}\right)\right),
\end{aligned}
$$

where for purely technical reasons we include an internal ordering $\theta_{a_{2}>a_{1}}$ for the already symmetric integrand. See ref. [14] for definition of the NLO 2-gluon function $\beta_{1 B}\left(k_{1}, k_{2}\right)$. We call sometimes the resulting MC weight the NLO short range correlation function, because it contributes significantly only if both gluons are non-soft and have transverse momenta (or rapidities) almost equal, as seen in the plot of Fig. 2. BE symmetrization requires clever reorganization of combinatorics, if want to gain on the computation speed by means of excluding terms equal zero from the BE symmetrization sum. For instance, BE symmetrization over 3 ! permutations of 3 arguments of a single NLO correlation function and one LO spectator distribution reduces to only 2 terms $^{7}$ :

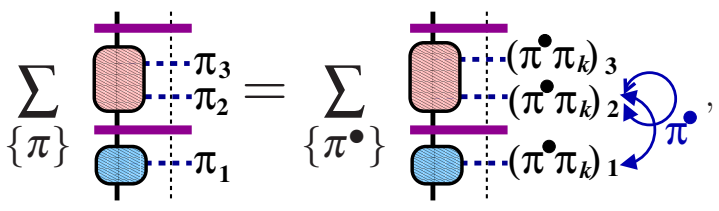

\footnotetext{
${ }^{7}$ Drawing of half of the ladder
} 
Permutation $\pi_{k}$ performs the right ordering $a_{\left(\pi_{k}\right)_{3}}>a_{\left(\pi_{k}\right)_{2}}>a_{\left(\pi_{k}\right)_{3}}$ at a given phase space point $k=\left(k_{1}, k_{2}, k_{3}\right)$. This is denoted in the above by $\left\{\pi^{\bullet}\right\}=\{(123),(213)\}$, where (123) is identity and (213) interchanges $\left(\pi_{k}\right)_{1}$ and $\left(\pi_{k}\right)_{2}$. Generalization to double insertion of NLO correlation function in the ladder in a sketchy graphical form is:

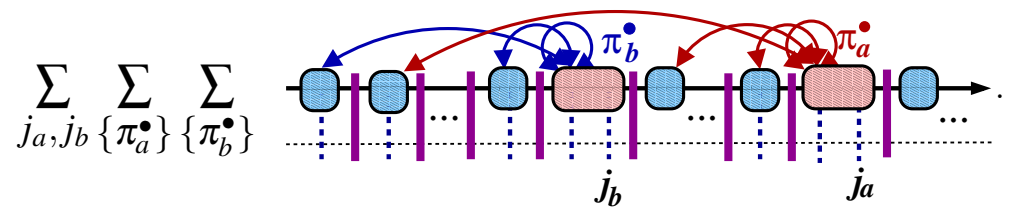

The corresponding algebraic formula of the above NLO ePDF can be found in ref. [14], together with the numerical result of the precision $\mathrm{MC}$ test.

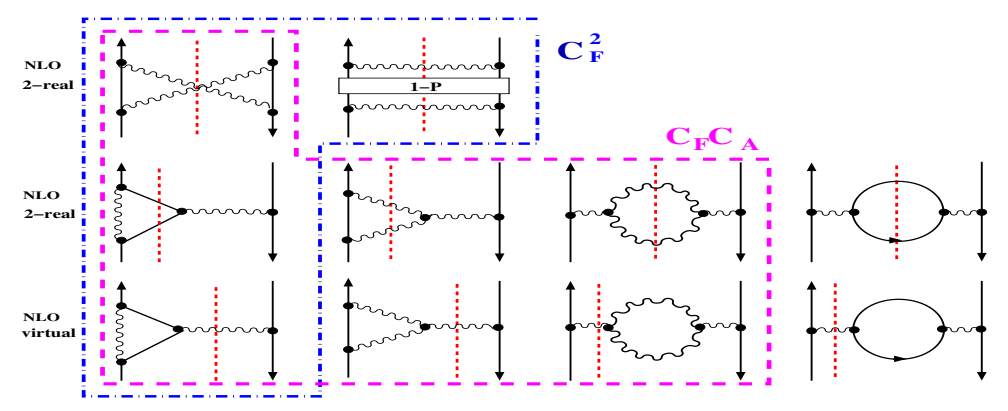

Figure 3: Feynman diagrams contributing to NLO non-singlet evolution.

So far we have considered the $\sim C_{F}^{2}$ diagrams, in Fig. 3. The remaining $\sim C_{F} C_{A}$ diagrams add new problem: strong cancellations between real and virtual contributions in the NLO correction ${ }^{8}$ due to final state radiation (FSR) Sudakov double log, This enforces exponentiation of the FSR already in the LO basic $\mathrm{MC}$, if we aim at positive weight MC events. In the basic MC each gluon in the LO ladder is replaced by

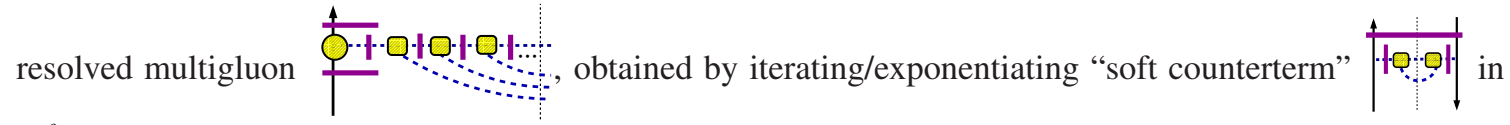
$K^{f}$. BE symmetrization is also done for FSR gluons, such that the complete NLO correcting weight includes the following sum:
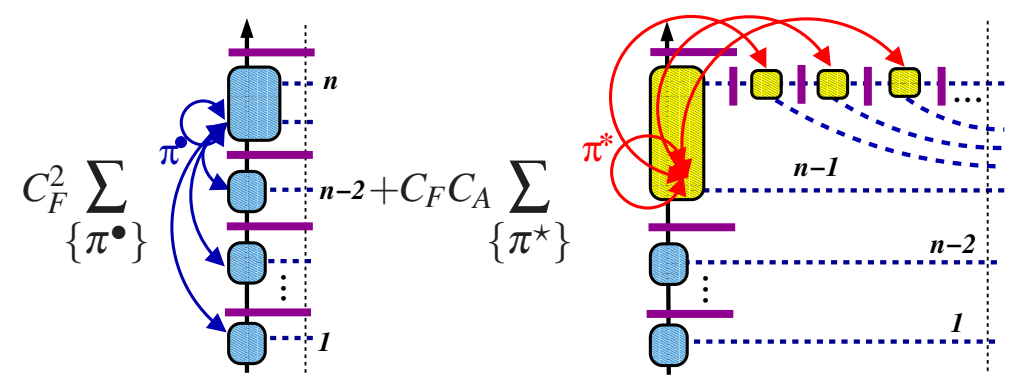

where $K^{f}=$ Th leading FSR Sudakov double log part is subtracted in $K^{f}$. The above sum in the MC weight was already tested for single NLO insertion, giving rise to a well behaved weight distribution. More testing is under way.

Summarizing, we report here on a small but essential part of the larger project, with the aim of implementing NLO DGLAP evolution of the parton distributions in fully unintegrated inclusive form in the

\footnotetext{
${ }^{8}$ Soft limit of this object was analyzed in ref. [15], exposing colour coherence effects.
} 
Monte Carlo with positive weights (weights equal one). The main result of the ongoing study, so far limited to nonsinglet ePDF, is that this is feasible. Once completed, this project will lead to new type of the parton shower MC for the initial state in LHC and other colliders with hadron beams.

\section{Acknowledgements}

Two of the authors (S.J and M.S.) wish to thank Theory Unit of Physics Division, CERN, for generous support during preparation of this work.

\section{References}

[1] L.N. Lipatov, Sov. J. Nucl. Phys. 20 (1975) 95;

V.N. Gribov and L.N. Lipatov, Sov. J. Nucl. Phys. 15 (1972) 438;

G. Altarelli and G. Parisi, Nucl. Phys. 126 (1977) 298;

Yu. L. Dokshitzer, Sov. Phys. JETP 46 (1977) 64.

[2] G. Curci, W. Furmanski, and R. Petronzio, Nucl. Phys. B175 (1980) 27.

[3] R. K. Ellis, H. Georgi, M. Machacek, H. D. Politzer, and G. G. Ross, Phys. Lett. B78 (1978) 281.

[4] J. C. Collins, D. E. Soper, and G. Sterman, Nucl. Phys. B250 (1985) 199.

[5] G. T. Bodwin, Phys. Rev. D31 (1985) 2616.

[6] T. Sjostrand, Phys. Lett. B157 (1985) 321.

[7] G. Marchesini and B. R. Webber, Nucl. Phys. B310 (1988) 461.

[8] H. Tanaka, Prog. Theor. Phys. 110 (2003) 963-973.

[9] J. C. Collins and F. Hautmann, JHEP 03 (2001) 016, hep-ph/ 0009286.

[10] J. C. Collins, T. C. Rogers, and A. M. Stasto, Phys. Rev. D77 (2008) 085009, 0708 . 2833.

[11] S. Frixione and B. R. Webber, JHEP 06 (2002) 029, hep-ph/ 0204244.

[12] D. R. Yennie, S. Frautschi, and H. Suura, Ann. Phys. (NY) 13 (1961) 379.

[13] J. C. Collins, Phys. Rev. D58 (1998) 094002, hep-ph/ 9806259.

[14] S. Jadach and M. Skrzypek, 0905.1399.

[15] M. Slawinska and A. Kusina, 0905.1403. 